\title{
Biomechanical and Morphological Differences between the Sclera Canal Ring and a Peripheral Sclera Ring in the Porcine Eye
}

\author{
Naim Terai ${ }^{\mathrm{a}} \quad$ Ursula Schlötzer-Schrehardt ${ }^{\mathrm{b}} \quad$ Eberhard Spoerl ${ }^{\mathrm{a}}$ \\ Karin Hornykewycz ${ }^{a}$ Janek Haentzschel ${ }^{a}$ Michael Haustein ${ }^{a}$ Lutz E. Pillunat ${ }^{a}$ \\ a Department of Ophthalmology, Carl Gustav Carus University Hospital, Dresden, and \\ ${ }^{b}$ Department of Ophthalmology, University of Erlangen-Nürnberg, Erlangen, Germany
}

\section{Key Words}

Sclera canal ring $\cdot$ Biomechanics $\cdot$ Hysteresis $\cdot$ Optic nerve head $\cdot$ Glaucoma

\begin{abstract}
Aim: To investigate a possible association between the biomechanical load and unload behaviour and the elastin content of the sclera canal ring (SCR) and a superiorly localized sclera ring (SPS) in the porcine eye. Methods: Two sclera rings were trephined from each of 40 porcine eyes, one containing the SCR and the other an SPS. The load and the unload curves were measured in the extension range of 0-2.0 $\mathrm{mm}$ by a biomaterial tester. Hysteresis was determined from the area enclosed by the loading and unloading curve. Histochemical staining with resorcin-fuchsin and morphometric analysis of paraffin-embedded sections of both rings were performed to detect the area occupied by elastin fibres. Results: At $1 \mathrm{~mm}$ extension, the mean load of the SCR was $0.89 \pm 0.22 \mathrm{~N}$ and that of the SPS $1.13 \pm 0.19 \mathrm{~N}$, which was not significantly different between both rings $(p>0.05)$. Mean hysteresis in the SCR was $1.55 \pm 0.30 \mathrm{~N} \times \mathrm{mm}$ and $1.90 \pm 0.18 \mathrm{~N} \times \mathrm{mm}$ in the SPS, which was significantly different between both rings $(p=0.01)$. Mean sclera thickness was $986 \mu \mathrm{m}$ in the SCR (range: 900-1,060 $\mu \mathrm{m}$ ) and $971 \mu \mathrm{m}$ in the SPS (range: 800-1,200 $\mu \mathrm{m}$ ) without a statistically significant difference between both sclera rings $(p=0.78)$. The area occupied by elastin fibres was $15.5 \pm 3.4 \%$ in the SCR
\end{abstract}

and $4.5 \pm 1.5 \%$ in the SPS, which was significantly different between both rings ( $p=0.0001)$. Conclusion: Hysteresis in the SCR was significantly lower than in the SPS, indicating a higher elasticity of the SCR in the porcine eye. This effect could be explained by a higher content of elastin in the surrounding ring of the peripapillary optic nerve head providing reversible contraction in cases of intra-ocular pressure variations.

Copyright $\odot 2011$ S. Karger AG, Basel

\section{Introduction}

The sclera forms the principal load-bearing tissue of the eye. Biomechanical effects of the sclera are mainly determined by 2 factors, the anatomy of the sclera (e.g. sclera thickness) and sclera tissue properties (sclera stiffness, sclera elasticity) [1-3]. Both features contribute to the biomechanical response of the sclera to intra-ocular pressure (IOP) and are therefore of particular interest in understanding the role of IOP in the pathogenesis of glaucoma, since scleral deformations due to IOP are directly transmitted to the tissues of the optic nerve head $(\mathrm{ONH})$, including the lamina cribrosa, the potential site of glaucomatous damage $[4,5]$.

Elastin, which is a complex polypeptide, seems to be involved in elasticity changes of the sclera as it is capable of resisting tissue deformation and of returning to its nor-

\section{KARGER}

Fax +4161306 1234 E-Mail karger@karger.ch www.karger.com

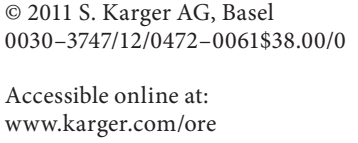

Naim Terai, MD

Department of Ophthalmology, Carl Gustav Carus University Hospital

Fetscherstrasse 74

DE-01307 Dresden (Germany)

Tel.+49 351458 5091, E-Mail naim.terai@ uniklinikum-dresden.de 
Fig. 1. SCR (blue arrow below; colours in the online version only) and SPS (red arrow above). The SPS (red circle above) was removed $2 \mathrm{~mm}$ above the SCR rim (blue circle below) using a 7-mm trepan.

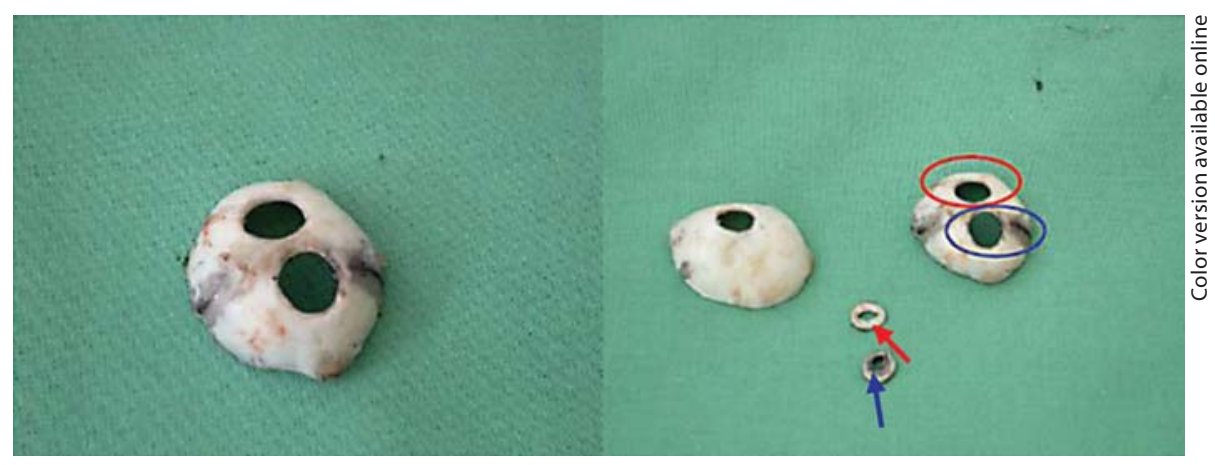

mal shape at low levels of stress [6]. Experimental studies by electron microscopy showed a large amount of elastin in the $\mathrm{ONH}$ region which was denser in the peripapillary sclera than in the sclera distant from the nerve. Elastin fibres were arranged in a dense circumferential configuration around the $\mathrm{ONH}$. In glaucomatous eyes, elastin, but also the relationship of elastin fibres to visible collagen, was changed. Elastin fibrils appeared wavy even though the density of elastin fibres seemed unchanged in glaucomatous eyes $[7,8]$.

A recently published study investigating the presence and distribution of elastin in the posterior regions of the mouse eye also showed that elastin in the sclera was densest in a ring surrounding the peripapillary $\mathrm{ONH}$, suggesting that this ring might provide a mechanism for reversible expansion and contraction when variations in IOP occur [9].

In accordance to these histological study results, the aim of the present study was to investigate whether the elastin-containing sclera canal ring (SCR) also shows a functional and morphological correlation to changes of stiffness and contraction, and secondly, if there are biomechanical differences compared to a more superiorly localized sclera ring (SPS).

\section{Methods}

Forty porcine eyes were examined. The eyes were cleaned of all extra-ocular structures. At the equator, the eyes were bisected horizontally, and the vitreous, retina and choroid were removed. Two sclera rings from each eye were cut by a trephine, one containing the SCR and the other an SPS which was removed $2 \mathrm{~mm}$ above the SCR rim. Both rings were excised by a $7-\mathrm{mm}$ trephine. The inner circle of each ring was removed using a $4-\mathrm{mm}$ trephine (fig. 1).

\section{Load-Extension Measurements}

In order to preserve the ring structure and the elastin continuity of the sclera rings, a modified circumferential measurement

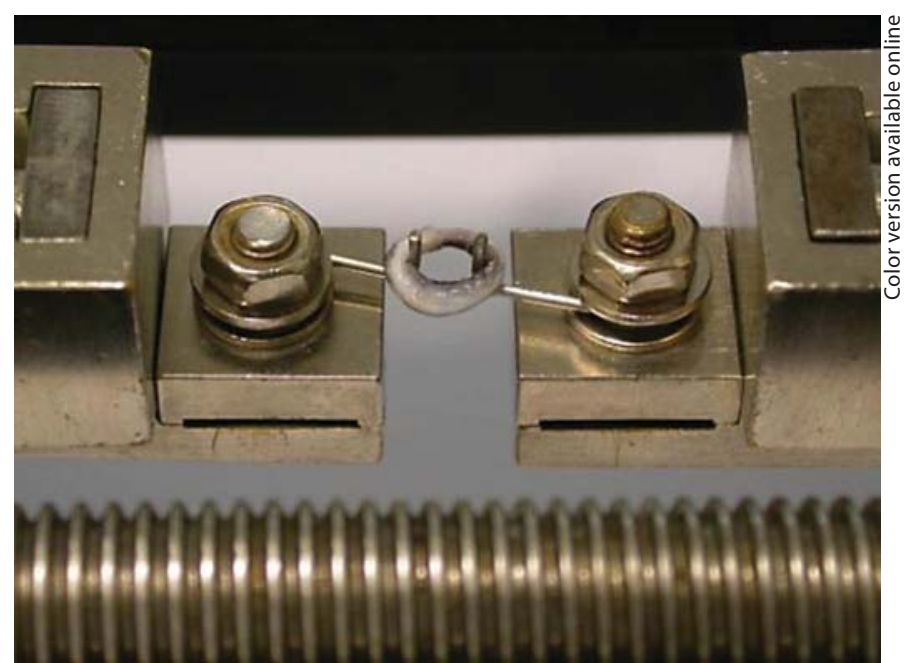

Fig. 2. Biomaterial tester with a sclera ring placed on 2 pins.

technique according to Seliktar et al. [10], Lillie et al. [11] and Lillie and Gosline [12] was chosen. Briefly, SCR and SPS were placed horizontally with a distance of $3 \mathrm{~mm}$ on 2 pins of the microcomputer-controlled biomaterial tester (MiniMat; Rheonetric Scientific $\mathrm{GmbH}$, Benshaim, Germany) at room temperature $\left(22^{\circ} \mathrm{C}\right.$; fig. 2). To tighten the rings, load-extension measurements were performed with a preload of $20 \mathrm{mN}$ which was applied for $2 \mathrm{~min}$. Then, the extension was increased linearly at a rate of $2 \mathrm{~mm} / \mathrm{min}$, and the load was detected. The end point of tissue displacement was $2 \mathrm{~mm}$. The load-extension curves were evaluated. The unload curves of the same rings were determined by extension release with the same velocity immediately after the load-extension curve.

Measurement of the tissue thickness was performed at 4 points of the sclera rings using an ultrasonic pachymeter (Pachy-Pen XL; Mentor, Norwell, Mass., USA).

\section{Histomorphometric Analysis of Elastin Fibres Stained with}

Resorcin-Fuchsin

Five-micrometre-thick tangential paraffin sections of SCR and SPS were stained with Weigert's resorcin-fuchsin according to standard protocols [13]. Sections were analysed with a light mi- 
Fig. 3. Histomorphometric analysis of elastin fibres stained with resorcin-fuchsin in the SCR $(\mathbf{a}, \mathbf{b})$ and in the SPS $(\mathbf{c}, \mathbf{d})$. a, c Light-microscopic appearance of a representative area of paraffin sections analysed for elastin content with purplestained elastin fibres. Resorcin-fuchsin. Magnification $\times 100$. c, $\mathbf{d}$ The area covered by elastin fibres was detected with an image-analysing programme by threshold analysis of grey values coded in red colour; elastin fibres (in red in the online version) were detected by threshold setting including the dark fibres and excluding the bright background.
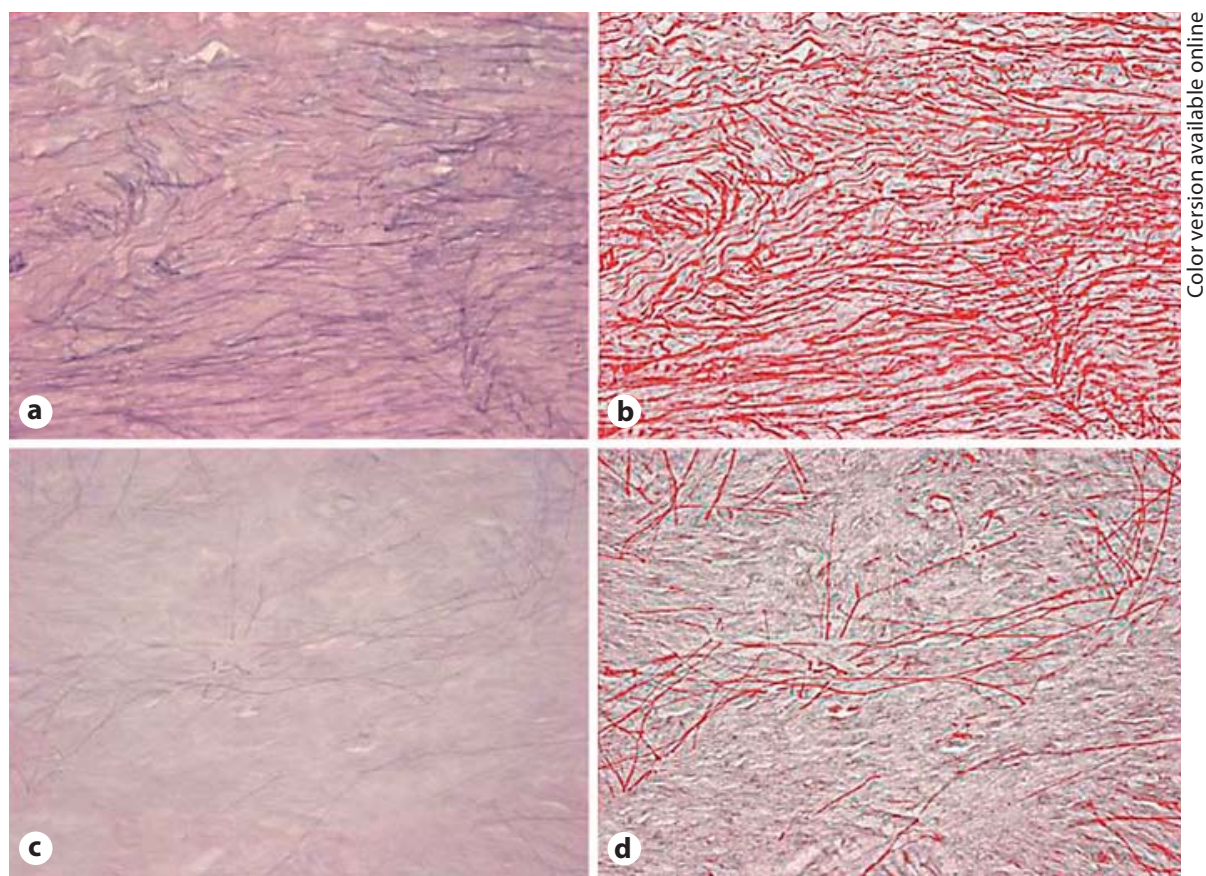

croscope (BX51; Olympus Optical Co., Hamburg, Germany), and digital pictures were taken from 5 different areas around the entire circumference of each ring with a CCD camera (F-View II; Soft Imaging System, Münster, Germany) at a magnification of $\times 40$ covering an area of $0.095 \mathrm{~mm}^{2}$. The percentage area occupied by elastin fibres in relation to the whole area analysed was detected with an image-analysing programme (Cell^F; Olympus Deutschland $\mathrm{GmbH}$, Hamburg, Germany) by threshold analysis of grey values; elastin fibres were detected by threshold setting including the dark fibres and excluding the bright background (fig. 3).

\section{Statistical Analyses}

The load and unload data for an extension of $1 \mathrm{~mm}$ were compared between SCR and SPS specimens by using Student's t test for paired samples. Mean hysteresis was determined from the area enclosed by the loading and unloading curves by calculating the number of pixels between the hysteresis loops. The amount of pixels was then converted to definite hysteresis values (newtons $\times$ millimetres). Statistical differences in the elastin content between both rings were determined by the unpaired Student's t test. A p value $<0.05$ was considered as statistically significant. All statistical analyses were performed using SPSS 15.0 .

\section{Results}

At $1 \mathrm{~mm}$ extension, the mean load of the SCR was 0.89 $\pm 0.22 \mathrm{~N}$ and that of the SPS $1.13 \pm 0.19 \mathrm{~N}$, which was not significantly different between both rings $(p>0.05$; fig. 4).

Sclera Canal Ring and Peripheral Sclera Ring in the Porcine Eye
Mean hysteresis was $1.55 \pm 0.30 \mathrm{~N} \times \mathrm{mm}$ in the SCR and $1.90 \pm 0.18 \mathrm{~N} \times \mathrm{mm}$ in the SPS, which was significantly different between both specimens $(p=0.01)$.

Sclera thickness ranged between 900 and $1,060 \mu \mathrm{m}$ in the SCR (mean: $986 \mu \mathrm{m}$ ) and between 800 and 1,200 $\mu \mathrm{m}$ (mean: $971 \mu \mathrm{m}$ ) in the SPS without a statistically significant difference between both sclera rings $(\mathrm{p}=0.78)$.

By light microscopy, purple-stained elastin fibres were arranged in a dense circumferential pattern in the SCR, mostly running in parallel, whereas they were rather sparsely distributed in the SPS without any preferred direction (fig. 3). The area occupied by elastin fibres was $15.5 \pm 3.4 \%$ in the SCR and $4.5 \pm 1.5 \%$ in the SPS of the total area analysed. This difference in elastin content between both rings was statistically significant $(\mathrm{p}=0.0001)$.

\section{Discussion}

The present study showed that the elastic hysteresis, defined as the lagging between the application of load and the removal of extension, was about $20 \%$ lower in the SCR than in the SPS, indicating a higher elasticity in the SCR than in the adjacent part of the sclera. This biomechanical finding was consistent with histomorphometric results showing a significantly higher elastin fibre content in the SCR than in the SPS. However, the load curve 


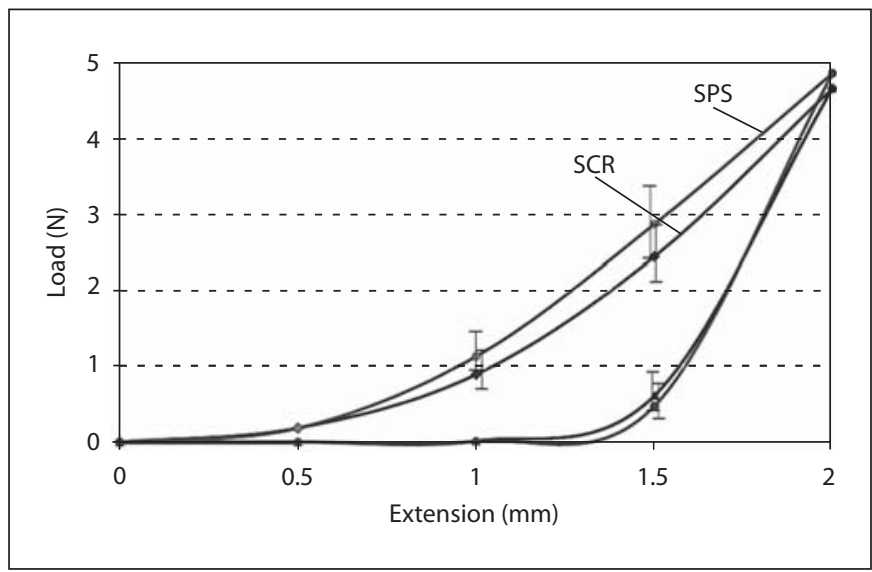

Fig. 4. Load-unload curves of the SCR and the peripheral SPS. Hysteresis was determined from the area enclosed by the loading and unloading curve.

in the SCR and SPS was without a statistically significant difference, indicating a nearly equal stiffness of both rings.

The results of the present study can be extrapolated to recent findings of Gelman et al. [9], in which the elastin content in the sclera was densest in a ring, consisting of a group of 10-12 elastin fibres and surrounding the peripapillary $\mathrm{ONH}$. These elastin fibres which were detected by immunohistochemistry and transmission electron microscopy corresponded to the directions of typical mechanical stresses [9]. The authors concluded that this ring might provide a mechanism for reversible expansion and contraction to possible IOP variations. The SCR in our study was chosen accordingly and contained the elastic peripapillary ring mentioned above which was confirmed by histochemical elastin staining and quantitative analysis of elastin fibres. Interestingly, in the study mentioned above, the amount of scleral elastin decreased with increasing distance from the $\mathrm{ONH}$ which is in agreement with findings of our study - the elasticity and the elastin content of the more superiorly localized SPS (with a distance of $2 \mathrm{~mm}$ above the SCR rim) was significantly lower, indicating a less flexible sclera in this region and suggesting a direct correlation of histological and biomechanical findings in the SCR.

Biomechanical effects of the sclera are not only determined by the sclera tissue properties like the stiffness, but also by the anatomy of the sclera, e.g. the sclera thickness. Sclera thickness in our specimens ranged between 900 and 1,060 $\mu \mathrm{m}$ in the SCR (mean: $986 \mu \mathrm{m}$ ) and between 800 and 1,200 $\mu \mathrm{m}$ (mean: $971 \mu \mathrm{m}$ ) in the SPS without a statistically significant difference between both sclera regions. However, a recent study by Elsheikh et al. [14] in human donor eyes, investigating the variation in thickness and biomechanical properties between different regions of the human sclera, observed a significant thickness shift from the maximum at the posterior pole to the minimum close to the equator, and increasing again towards the limbus. With reference to specific stress levels, the scleral stiffness showed a progression from the posterior region towards the limbus, suggesting that the posterior region's low mechanical stiffness is partly balanced by a large thickness, giving the area surrounding the lamina cribrosa and the $\mathrm{ONH}$ more adequate resistance to loading [14]. In accordance to our study, sclera thickness was also obtained by an ultrasound pachymeter so that methodological differences can be ruled out. Repeated measurements of sclera thickness in our study were performed by two experienced investigators (E.S., N.T.) so that possible measurement bias and the subjective influence of a single investigator can be excluded. However, in contrast to our study, thickness measurements in the study mentioned above were carried out along 8 meridian lines of strips extending from the posterior pole to the limbus and not exclusively from rings of the sclera or the sclera canal so that differences in location but also in geometrical configuration of the specimens might explain the observed differences in sclera thickness. Given that measurements in our study were performed accurately, the effect of sclera thickness, as a possible confounding factor, on the biomechanical stiffness and elasticity behaviour can be ruled out as no significant difference between thickness of the SCR and the SPS was observed. Against this background, our results indicate even more that the biomechanical behaviour of the SCR is not (only) explained solely by the thickness of the sclera, but also by other factors like the elastin distribution or elastin content in this region of the sclera. To date, some previous studies already showed that alterations of the elastin network in the surrounding connective tissue of the $\mathrm{ONH}$ were associated with glaucoma pathogenesis and that damage could affect the ability of the $\mathrm{ONH}$ to withstand deformation by compromising the mechanical stiffness of the tissue [15-19]. Despite histologically different elastin distributions and different elasticity responses, it remains unclear why the stiffness, measured by the load curve, did not also reveal significant differences between the two rings. The difference in the circumferential mechanical behaviour of the SCR and the SPS might be caused by the scleral morphology in two different rings, predominantly by the elastin distribution. As mentioned 
before, elastin stretches under stress but returns to its original length after release of tension. Collagen, however, is capable of load bearing up to a certain level but is not able to restore its original position as fast as elastin does [6]. Elastin might therefore play a more important role in the process of contraction than in the process of expansion, indicating that the SCR with its higher elastin content might have a stronger impact on contraction than the SPS. Interestingly, 2 experimental studies of Quigley et al. $[7,8]$ showed that the elastin fibres in glaucomatous eyes appeared wavy and seemed to be separated from other elements in disorganized laminar beams, including a decrease in collagen density. They hypothesized that elastin, which is analogous to a spring normally stretching in a straight line between 2 fixed points, loses its fixed framework leading to a curled shape of the elastin fibres. At this point it is conceivable that changes in elastin architecture might also lead to altered biomechanical responses of the tissues, despite an unchanged elastin density in glaucomatous eyes. Further morphological and biomechanical data are certainly needed to investigate this issue in more detail, especially in diabetic, myopic and glaucomatous eyes.

The present study has two major limitations. Firstly, uniaxial loading is different from biaxial loading existing in intact eye globes. Secondly, it has been suggested that load-extension relationships from extensometry are considerably stiffer than those derived using inflation tests [20].

To the best of our knowledge for the first time in the present study extensometry measurements were performed on sclera rings and not on strips in order to preserve the typical geometric ring configuration and not to interrupt the continuity of the elastin-collagen network. It can only be assumed that the biomechanical loading and unloading behaviour is different compared to strips where straightening occurs as strips originate from a spherical curved form and biomechanical alterations might be more advanced than in the natural ring form of the sclera [20].

Taking all findings together, the results of the present study showed that the elasticity was higher (or the hysteresis lower) in the SCR specimens of the porcine eye compared to a more superiorly localized SPS, which was consistent with a histologically higher elastin content in the SCR than in the SPS. This effect was not dependent on the sclera thickness of the tissue, indicating that elastin has a modulating biomechanical effect in the SCR, which cannot be explained by sclera thickness only.

\section{References}

1 Sigal IA, Flanagan JG, Ethier CR: Factors influencing optic nerve head biomechanics. Invest Ophthalmol Vis Sci 2005;46:41894199.

2 Sigal IA, Ethier CR: Biomechanics of the optic nerve head. Exp Eye Res 2009;88:799807.

3 Norman RE, et al: Dimensions of the human sclera: thickness measurement and regional changes with axial length. Exp Eye Res 2010; 90:277-284

4 Bellezza AJ, Hart RT, Burgoyne CF: The optic nerve head as a biomechanical structure: initial finite element modeling. Invest Ophthalmol Vis Sci 2000;41:2991-3000.

5 Downs JC, Roberts MD, Burgoyne CF: Mechanical environment of the optic nerve head in glaucoma. Optom Vision Sci 2008; 85:425-435.

6 Watson PG, Young RD: Scleral structure, organisation and disease: a review. Exp Eye Res 2004;78:609-623.

7 Quigley HA, Brown A, Dorman-Pease ME: Alterations in elastin of the optic nerve head in human and experimental glaucoma. $\mathrm{Br} \mathrm{J}$ Ophthalmol 1991;75:552-557.
8 Quigley HA, Dorman-Pease ME, Brown AE: Quantitive study of collagen and elastin of the optic nerve head and sclera in human and experimental monkey glaucoma. Curr Eye Res 1991;10:877-888.

9 Gelman S, et al: The presence and distribution of elastin in the posterior and retrobulbar regions of the mouse eye. Exp Eye Res 2010;90:210-215.

10 Seliktar D, et al: Dynamic mechanical conditioning of collagen-gel blood vessel constructs induces remodeling in vitro. Ann Biomed Eng 2000;28:351-362.

11 Lillie MA, Chalmers GW, Gosline JM: The effects of heating on the mechanical properties of arterial elastin. Connect Tissue Res 1994;31:23-35.

12 Lillie MA, Gosline JM: Limits to the durability of arterial elastic tissue. Biomaterials 2007;28:2021-2031.

13 Clark G: Staining Procedures, ed 3. Baltimore, Williams \& Wilkins, 1973, p 60.
14 Elsheikh A, et al: Regional variation in the biomechanical properties of the human sclera. Exp Eye Res 2010;90:624-633.

15 Pena JD, et al: Increased elastin expression in astrocytes of the lamina cribrosa in response to elevated intraocular pressure. Invest Ophthalmol Vis Sci 2001;42:2303-2314.

16 Pena JD, et al: Elastosis of the lamina cribrosa in glaucomatous optic neuropathy. Exp Eye Res 1998;67:517-524.

17 Hernandez MR, Ye H: Glaucoma: changes in extracellular matrix in the optic nerve head. Ann Med 1993;25:309-315.

18 Hernandez MR: Ultrastructural immunocytochemical analysis of elastin in the human lamina cribrosa: changes in elastic fibers in primary open-angle glaucoma. Invest Ophthalmol Vis Sci 1992;33:2891-2903.

19 Hernandez MR, et al: Age-related changes in the extracellular matrix of the human optic nerve head. Am J Ophthalmol 1989;107:476484

20 Elsheikh A, Anderson K: Comparative study of corneal strip extensometry and inflation tests. J R Soc Interface 2005;2:177-185. 